\title{
Comparison between Conventional \\ Unenhanced and Virtual Unenhanced Imaging of Hepatopancreaticobiliary System with Third-Generation Dual-Source Dual-Energy CT-An Observational Study
}

\author{
Swathigha Selvaraj ${ }^{1}$ Niyas N.P. ${ }^{1} \quad$ Rupa Renganathan ${ }^{1}$ Rajkumar Ramasamy ${ }^{1}$ \\ Rinoy Ram Anandan ${ }^{1}$ Venkatesh Kasi Arunachalam¹ Mathew Cherian ${ }^{1}$ \\ ${ }^{1}$ Department of Radiology, Kovai Medical Center and Hospital, \\ Address for correspondence Venkatesh Kasi Arunachalam, \\ Avanashi Road, Coimbatore, India \\ DMRD, DNB, FRCR, Department of Radiology, Kovai Medical \\ Center and Hospital, Avanashi Road, Coimbatore 641014, India \\ (e-mail: drkasivenkatesh@yahoo.co.in).
}

J Gastrointestinal Abdominal Radiol ISGAR 2021;4:229-235.

\begin{abstract}
Objectives The aims of our study were to assess the comparability of conventional unenhanced images (CUIs) of hepatopancreaticobiliary system with virtual unenhanced images (VUIs) derived from arterial and portal venous phases acquired in a third-generation, dual-source, dual-energy CT (DECT), and also to assess the best dataset among these VUIs. We also calculated the radiation effective dose (ED) reduction by eliminating noncontrast acquisition.

Materials and Methods 60 patients were included in our study. Unenhanced images in single energy and contrast-enhanced images in dual-energy mode were acquired. Arterial virtual unenhanced (AVU) and portal virtual unenhanced (PVU) images were generated and compared with CUI, using both objective and subjective methods. The ED was calculated separately for each phase. Statistical significance between difference in mean attenuation values were analyzed using ANOVA and unpaired student t-test.

Results In our study, the difference in mean attenuation of liver, spleen, and pan-

Keywords

- True unenhanced

- Virtual unenhanced

- Third generation

- Dual source

- Dual energy

- Radiation dose creas between the three phases-CU, AVU, and PVU-were insignificant with $p$-value $>0.05$. This indicates that the values were comparable. Among the VUI, AVU images were statistically superior in image quality. Elimination of noncontrast CT from triple phase abdominal imaging can achieve an average ED reduction of $39 \%$.

Conclusions We conclude that VUI generated in third-generation, dual-source DECT has diagnostic image quality and can replace the CUI in triple-phase studies, with a mean ED reduction by $39 \%$. The VUI obtained from arterial phase is superior to those obtained from portal venous phase.
\end{abstract}

published online June 18,2021
DOI https://doi.org/ $10.1055 / \mathrm{s}-0041-1730095$ ISSN 2581-9933
(C) 2021. Indian Society of Gastrointestinal and Abdominal Radiology. This is an open access article published by Thieme under the terms of the Creative Commons Attribution-NonDerivative-NonCommercial-License, permitting copying and reproduction so long as the original work is given appropriate credit. Contents may not be used for commercial purposes, or adapted, remixed, transformed or built upon. (https://creativecommons.org/licenses/by-nc-nd/4.0/). Thieme Medical and Scientific Publishers Pvt. Ltd. A-12, 2nd Floor, Sector 2, Noida-201301 UP, India 


\section{Introduction}

Beginning from the era of single-slice, sequential CT scanner and advancing toward multislice spiral $\mathrm{CT}$, with ultrafast true isotropic volume imaging, the technology has undergone an enormous change over the last few decades. Conventional CT scanners which operate at single energy provide morphological information only, with little material specific information. This is overcome by the use of dual-energy techniques which use two X-ray spectra to obtain two sets of images and data, which are collected on the principle "different materials show different attenuation at different energy levels." Dual-energy CT (DECT) is also referred to as "spectral CT" because of the usage of two different energy spectra. ${ }^{1}$

Some of the uses of DECT in hepatopancreaticobiliary imaging include detection and staging of hypervascular liver lesions, such as hepatocellular carcinoma and hypervascular liver metastasis, which are more conspicuous on low-energy images (e.g., $80 \mathrm{kVp}$ ) than they are on high-energy images (e.g., $140 \mathrm{kVp}$ ) in the arterial phase of enhancement, determining the components of a hepatic lesion, which helps in characterizing the lesions, such as hepatic adenoma or hepatocellular carcinoma if fat is present within the lesion, differentiating simple cysts from hypovascular metastasis and fatty infiltration from hypoattenuating pancreatic masses and necrosis, with the help of water and iodine density images. ${ }^{2-5}$

A triple-phase study is used for characterization of liver lesions. By using DECT with the above advantages, it is possible to identify and characterize the lesions with greater accuracy. But there is a concern about the radiation dose. Radiation exposure required for DECT depends on the technology used. The aim is to use the same possible dose as would be used in a single-energy CT (SECT), so that additional information can be obtained without an increase in dose. The specific parameters influencing the radiation dose are tube current, pitch, and energy. If low-tube currents are used, dual-energy images may be obtained with radiation doses similar to those used to acquire single energy images. This dose reduction is further aided by replacing the conventional unenhanced images (CUI) by the virtual unenhanced images (VUI). ${ }^{6,7}$

VUI are a set of images which are obtained by using the technique of material decomposition into iodine, fat, and water. These images are generated by subtracting the iodine signal from contrast images, whereas iodine-only images are obtained by selectively choosing the contrast density material images ( - Fig. 1) ${ }^{8,9}$ For obtaining good VUI, effective separation of the two datasets is required. This can be achieved to an acceptable level with the new third-generation, dual-source DECT scanners, with the use of $100 \mathrm{kV}$ in one and $150 \mathrm{kV}$ in the other tube, thicker prefilter, and advanced modeled iterative reconstruction (ADMIRE) algorithm. ${ }^{10}$

The aim of our study was to assess whether VUI generated in third-generation, dual-source DECT can replace the CUI in studies done for hepatopancreaticobiliary system. The objectives include assessment of the comparability of CUI with VUI derived from arterial and portal venous phases by
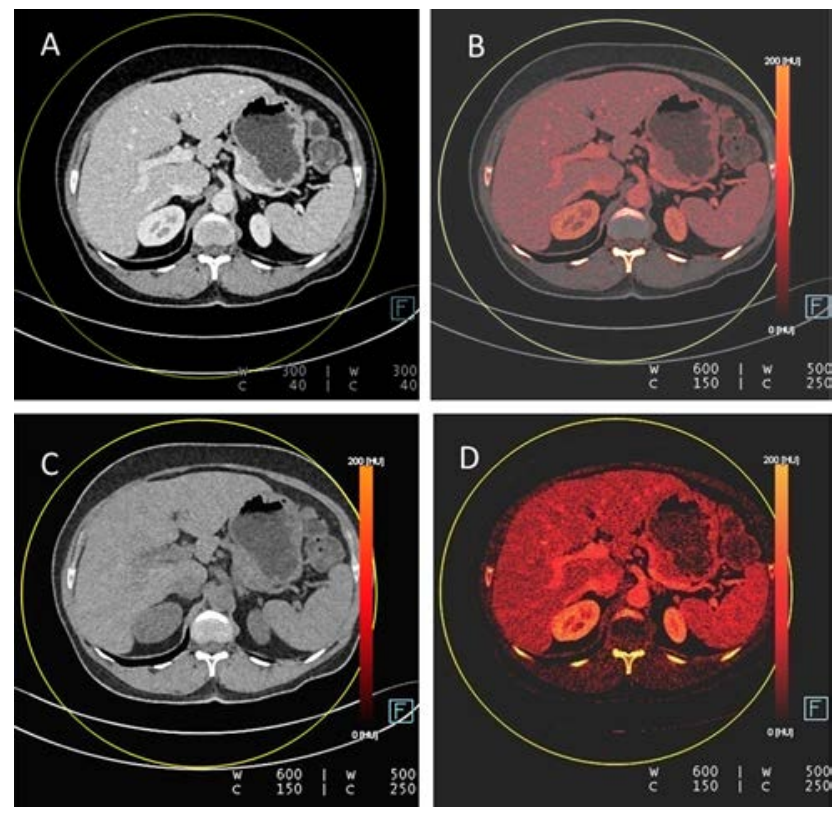

Fig. 1 (A) Axial contrast-enhanced portal venous phase image and the iodine color-coded image (B), Virtual unenhanced image (C) and iodine-only image (D) generated using liver virtual noncontrast (VNC) software from the portal venous dataset.

objective and subjective methods, identifying the best dataset among the arterial/portal venous VUI, and calculation of the percentage of radiation effective dose (ED) reduction by eliminating noncontrast acquisition.

\section{Materials and Methods}

\section{Patient Selection}

This study was approved by the local ethical and scientific committee, and a written informed consent was obtained from all participants. All patients who came to the radiology department for evaluation of the hepatopancreaticobiliary system and who were subjected to DECT between November 2018 to November 2019 with varied clinical profile such as cirrhosis, hepatocellular carcinoma, fatty liver, cholangiocarcinoma, metastasis, and liver lesion characterization were included in this study. The patients who were being evaluated as donor for liver transplant program/who underwent transarterial radioembolization (TARE) or transarterial chemoembolization (TACE) for hepatocellular carcinoma/metastatic lesions were excluded from the study.

\section{Methodology}

The study was done on a third-generation, dual-source DECT scanner (Somatom force, Siemens Healthcare).

CUI in single-energy mode and contrast-enhanced, dual-phase images (late arterial and portal venous) in dual-energy mode were acquired. Both sets were acquired with a pitch of 1 . In single-energy scan, the tube voltage used was $120 \mathrm{Kv}$. In dual-energy, tube A operates at $100 \mathrm{kVp}$ and tube $B$ at $150 \mathrm{kVp}$. The patient was placed in supine position with extended arms. 
We used Omnipaque $350 \mathrm{mg} / \mathrm{mL}$ as the contrast agent at a flow rate of $4 \mathrm{~mL} / \mathrm{s}$, followed by $20 \mathrm{~mL}$ saline flush, using an automated dual-syringe power injector through an 18-gauge intravenous (IV) access placed in the right median cubital vein. The volume of contrast was decided based on the weight of the patient. The contrast scan was performed by using a bolus tracking software application. Region of interest (ROI) was placed in the abdominal aorta and when Hounsfield units $(\mathrm{HU})$ of 150 was reached, the scan was triggered with a delay of 15 seconds for arterial phase and 60 seconds for portal venous phase. In conventional unenhanced (CU) and portal venous phases, the scan coverage was from the diaphragm to the level of the ischial tuberosities, and in the arterial phase, the scan coverage was to the iliac crests.

ADMIRE software, Siemens Healthcare, was used for reconstruction of images, and analysis was done in Syngo.via workstation, using the inbuilt Liver VNC software. VUI were generated from arterial and portal venous phase images.

\section{Image Analysis}

The images from each patient was separated into three sets, namely, CU, arterial virtual unenhanced (AVU), and portal venous virtual unenhanced (PVU) images. The objective image analysis was done by comparing the HU values, and the subjective image analysis was done using the five-point scale for anatomical details and artifacts.

Objective image analysis: The attenuation of liver, spleen, pancreas, portal vein, aorta, and lesion in all the three datasets were measured. $1 \mathrm{~cm}^{2}$ circular ROI in the liver, pancreas, spleen and larger lesions and $0.5 \mathrm{~cm}^{2} \mathrm{ROI}$ in the portal vein, aorta, calcifications and smaller lesions were placed, and the corresponding attenuation was measured. The ROI was placed in the liver and spleen at the level of portal vein bifurcation, and in the pancreas, portal vein and aorta at the level of portal vein formation. The maximum possible circular ROI was used for gallbladder calculus.

Subjective image analysis: This scoring criteria was developed on the basis of the previous studies done by Li et al and De Ceccoa et al as shown in - Table $1 .^{11,12}$

Table 1 Subjective image analysis criteria based on artifacts and anatomical details

\begin{tabular}{|c|c|c|}
\hline Grade & Artifacts & $\begin{array}{l}\text { Anatomical details and } \\
\text { lesions }\end{array}$ \\
\hline 1 & Severe artifacts & $\begin{array}{l}\text { Organs-not assessable, } \\
\text { lesions-not visible }\end{array}$ \\
\hline II & $\begin{array}{l}\text { Artifacts affecting diag- } \\
\text { nostic information }\end{array}$ & $\begin{array}{l}\text { Anatomical details of } \\
\text { organs-not clearly visible, } \\
\text { lesions-not clear }\end{array}$ \\
\hline III & $\begin{array}{l}\text { Obvious artifacts but } \\
\text { acceptable }\end{array}$ & $\begin{array}{l}\text { Anatomical details of } \\
\text { organs and lesions-seen to } \\
\text { an acceptable extent }\end{array}$ \\
\hline IV & $\begin{array}{l}\text { Artifacts were seen in } \\
\text { other organs not inter- } \\
\text { fering with the diagnosis }\end{array}$ & $\begin{array}{l}\text { Organs and lesions-seen } \\
\text { clearly }\end{array}$ \\
\hline V & Minimum or no artifacts & $\begin{array}{l}\text { Organs and lesions-seen } \\
\text { clearly }\end{array}$ \\
\hline
\end{tabular}

\section{Statistical Analysis}

The results were collected in a Microsoft Excel file. Statistical Package for Social Sciences (SPSS) version 21was employed to analyze data. HU measurements were numeric; variables had a Gaussian distribution, so that mean values and standard deviation (SD) could be calculated. All measurements were expressed as the mean value \pm SD.

The difference in mean attenuation values between the CU, AVU and PVU groups were compared by ANOVA; $p$-value $<0.05$ was considered significant and $>0.05$ was considered insignificant.

The difference in mean attenuation values between the individual groups were compared in required areas, using unpaired student $t$-test, and $p$-value $<0.05$ was considered significant and $>0.05$ was considered insignificant.

The mean dose length product (DLP) was measured for CU phase, arterial enhanced phase and portal venous enhanced phase separately. The ED in mSv was calculated using the formula $E D=D L P \times k$, where $k$ is a constant and its value for abdomen and pelvis is 0.015 . The percentage of dose reduced by avoiding noncontrast scan was then calculated.

\section{Results}

About 60 patients who underwent dual energy, triple-phase $\mathrm{CT}$ imaging for proven or suspected lesion in hepatopancreaticobiliary system were included. Mean age of the sample group was 51.5 years, with range between 31 to 72 years. Among the 60 patients, males contributed to the major number, accounting for approximately $62 \%$ of the total, and females formed the rest of the total (38\%). Since all the patients underwent scanning for known or suspected hepatobiliary pathology, they presented with varied clinical profiles and diagnoses, among which cirrhosis was the most common proven/suspected diagnosis.

\section{Objective Criteria}

The $p$-value for difference in mean attenuation of liver, spleen, pancreas and gallbladder calculus size was insignificant. The attenuation values were comparable with no significant difference between CU, AVU, and PVU images (-Table 2). Similar results were obtained for both solid and cystic lesions, identified during the scan. The $p$-values for difference in mean attenuation of aorta, portal vein, inferior vena cava (IVC), and gallstone were significant and were not statistically comparable.

\section{Subjective Score}

The subjective score was analyzed using a five-point scale ranging from grade 1 to 5 , where 1 was the least and 5 was the best possible score. The mean subjective score obtained for AVU and PVU were $4.01 \pm 0.61$ and $3.7 \pm 0.46$ (mean \pm $\mathrm{SD})$, respectively. The $p$-value for individual differences of subjective score between AVU and PVU was 0.001, indicating that the difference in image quality was statistically significant and AVU images was superior among the two sets of VUI. 
Table 2 Mean attenuation and SD in conventional unenhanced and virtual unenhanced images and their p-values

\begin{tabular}{|c|c|c|c|c|c|c|}
\hline & & CU & AVU & PVU & $p$-value & Significance \\
\hline \multirow[t]{2}{*}{ Liver } & Mean & 54.05 & 50.98 & 50.73 & \multirow[t]{2}{*}{0.327} & \multirow[t]{2}{*}{ Insignificant } \\
\hline & SD & 11.05 & 11.15 & 11.31 & & \\
\hline \multirow[t]{2}{*}{ Spleen } & Mean & 46.67 & 45.91 & 45.42 & \multirow[t]{2}{*}{0.17} & \multirow[t]{2}{*}{ Insignificant } \\
\hline & SD & 2.54 & 3.9 & 3.82 & & \\
\hline \multirow[t]{2}{*}{ Pancreas } & Mean & 41.93 & 39.26 & 39.19 & \multirow[t]{2}{*}{0.168} & \multirow[t]{2}{*}{ Insignificant } \\
\hline & SD & 8.17 & 7.27 & 6.48 & & \\
\hline \multirow[t]{2}{*}{ Lesion } & Mean & 51.83 & 37.71 & 40.26 & \multirow[t]{2}{*}{0.862} & \multirow[t]{2}{*}{ Insignificant } \\
\hline & SD & 81.81 & 45.23 & 51.61 & & \\
\hline \multirow[t]{2}{*}{ Size of GB calculi } & Mean & 0.94 & 0.9 & 0.85 & \multirow[t]{2}{*}{0.984} & \multirow[t]{2}{*}{ Insignificant } \\
\hline & SD & 0.41 & 0.4 & 0.41 & & \\
\hline \multirow[t]{2}{*}{ GB calculus attenuation } & Mean & 818.29 & 383.86 & 376.57 & \multirow[t]{2}{*}{0.048} & \multirow[t]{2}{*}{ Significant } \\
\hline & SD & 488.96 & 290.36 & 243.75 & & \\
\hline \multirow[t]{2}{*}{ Aorta } & Mean & 44.31 & 34.71 & 33.71 & \multirow[t]{2}{*}{0.001} & \multirow[t]{2}{*}{ Significant } \\
\hline & SD & 5.86 & 7.5 & 6.72 & & \\
\hline \multirow[t]{2}{*}{ IVC } & Mean & 42.01 & 34.33 & 33.76 & 0.001 & \multirow[t]{2}{*}{ Significant } \\
\hline & SD & 5.66 & 6.34 & 6.29 & 6.39 & \\
\hline \multirow[t]{2}{*}{ Portal vein } & Mean & 36.59 & 34.78 & 33.86 & \multirow[t]{2}{*}{0.001} & \multirow[t]{2}{*}{ Significant } \\
\hline & SD & 6.34 & 6.29 & 6.39 & & \\
\hline
\end{tabular}

Abbreviations: AVU, arterial virtual unenhanced; CU, contrast unenhanced; GB, gallbladder; IVC, inferior vena cava; PVU, portal virtual unenhanced; SD, standard deviation.

Note: $p$-value $<0.05-$ significant

\section{Radiation Dose}

Mean DLP and effective dose were calculated ( - Table 3 ). It was found that there was a significant effective dose reduction of $39 \%$ when we removed the noncontrast unenhanced acquisition from the total examination and replaced them with virtual unenhanced images.

\section{Discussion}

In this modern era, with technological advancements, DECT by using different photon energies has gained worldwide acceptance, because of its material decomposition methods and promising potential in radiation dose reduction. It has immense advantages over single-energy CTs right from lesion detection, characterization, and differentiation. ${ }^{4}$

However, in the previously used first- and secondgeneration, dual-source CTs, certain disadvantages like poor spatial resolution, motion-related artifacts, increased noise and increased radiation dose existed and hence they did not become popular. ${ }^{13-16}$ These problems were addressed in the new third-generation, dual-source, dual-energy CT with the usage of ADMIRE algorithm as against the previously used filtered back projection (FBP). It was found that the lesion detection rate was increased to approximately $80 \%$ and dose reduction was achieved to approximately $41 \%$ when compared with the second-generation CT scanners. ${ }^{8}$ According to our study, it was found that the difference in mean attenuation of liver, spleen and pancreas between the three phases$\mathrm{CU}, \mathrm{AVU}$ and PVU images-were insignificant, with $p$-value > 0.05( - Fig. 2). This indicated that there was complete separation of iodine from these organs, and values were comparable with no significant difference between the attenuation values, even in patients with fatty infiltration of the liver. This is in agreement with De Ceccoa et al, in that there is no statistically significant difference in the mean attenuation values of the liver, spleen, and psoas muscles between CU, AVU and PVU images. ${ }^{12}$ Graser et al also found no statistical difference in mean attenuation values of the liver and renal parenchyma between CU and VUI derived from the postcontrast nephrographic phase dataset. ${ }^{17}$ In a study done by Durieux et al, they

Table 3 Mean and SD of DLP and radiation ED in conventional unenhanced and enhanced phases

\begin{tabular}{|l|l|l|l|l|}
\hline & \multicolumn{2}{|c|}{ DLP } & \multicolumn{3}{c|}{ Radiation ED } \\
\hline Phase & Mean & SD & Mean & SD \\
\hline Conventional unenhanced & 407.27 & 77.07 & 6.11 & 1.16 \\
\hline Arterial enhanced & 230.6 & 40.89 & 3.45 & 0.61 \\
\hline Portal venous enhanced & 423.18 & 72.97 & 6.33 & 1.11 \\
\hline
\end{tabular}

Abbreviations: DLP, dose length product; ED, effective dose; SD, standard deviation 

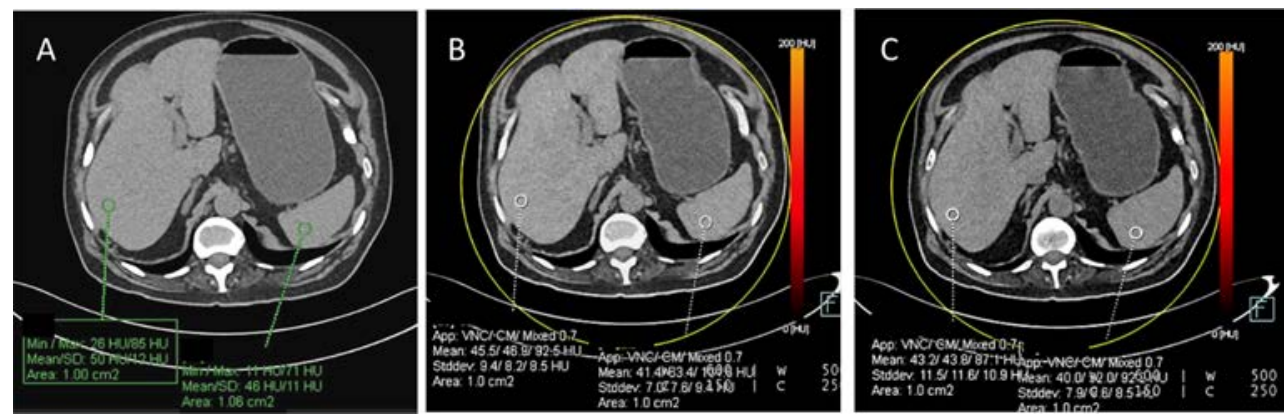

Fig. 2 Attenuation of liver and spleen in (A) noncontrast unenhanced image (B) arterial virtual unenhanced image (C) portal venous virtual unenhanced image.

observed no statistically significant difference between CU and PVU in the solid organs, including liver and spleen. ${ }^{18}$

In our study, the difference in mean attenuation of aorta, IVC and main portal vein (MPV) between the CUI and VUI were significant with $p$-value $<0.05$, indicating that the attenuation values are underestimated by VUI for these vessels ( - Fig. 3). In our study, we used a liver-specific algorithm, a three-material decomposition algorithm (iodine, fat, and water). The general algorithm uses a two-material decomposition algorithm (iodine and water). These algorithms affect the calculation of the VU datasets and the corresponding attenuation values. The underestimation of attenuation values in vessels in our study could be secondary to the three-material decomposition algorithms. This is in concordance with the study done by De Ceccoa et al which showed that statistically significant reduction in the mean attenuation was observed in the aorta. ${ }^{12}$ However, Laukamp et al concluded in their study that the attenuation of aorta and portal vein in AVU and PVU images showed no significant difference compared with $\mathrm{CU}$ images. ${ }^{19}$ The mean reduction in HU between the CUI and VUI were $\leq 10 \mathrm{HU}$ in all these vessels in both phases in our study. The clinical significance of this minimal attenuation difference is debatable.

Significant reduction of mean $\mathrm{HU}$ value with mild reduction in size of calcified gallbladder stones was noted in our study. The difference in $p$-value (0.048) was significant, indicating that they are not comparable. The mean HU values in VUI were underestimated when compared with CU images, indicating that calcium was subtracted along with iodine in VUI (-Fig. 4). However, there was no statistically significant difference in mean size of the gallbladder stones between the CU and VUI ( - Fig. 5). De Ceccoa et al quoted in their study that calcified liver lesions and gallbladder calculi showed a decrease in $\mathrm{HU}$, and small calculus were completely subtracted in the VUI. ${ }^{12}$ In another study done by Lee et al, they found out that calcium stones were underestimated in
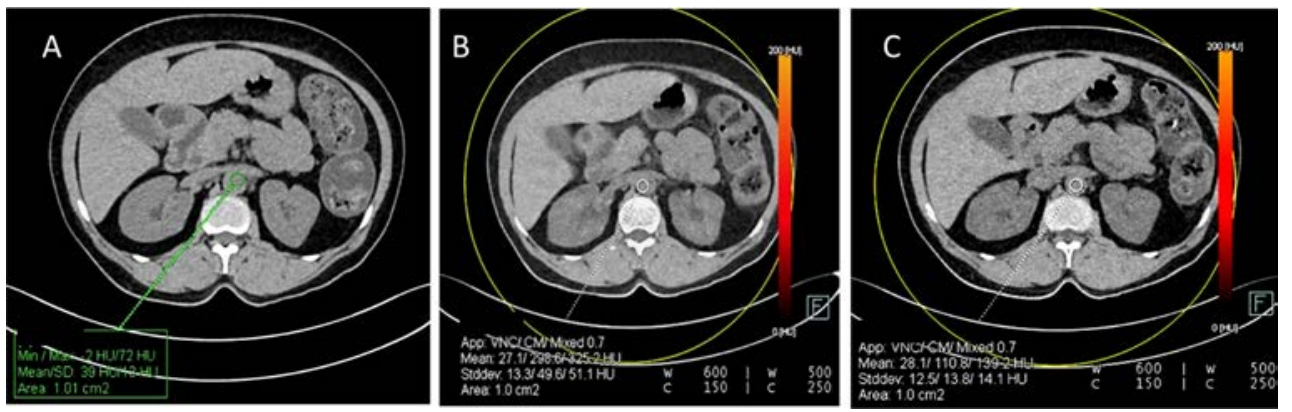

Fig. 3 Attenuation of aorta in (A) noncontrast unenhanced image (B) arterial virtual unenhanced image (C) portal venous virtual unenhanced image.
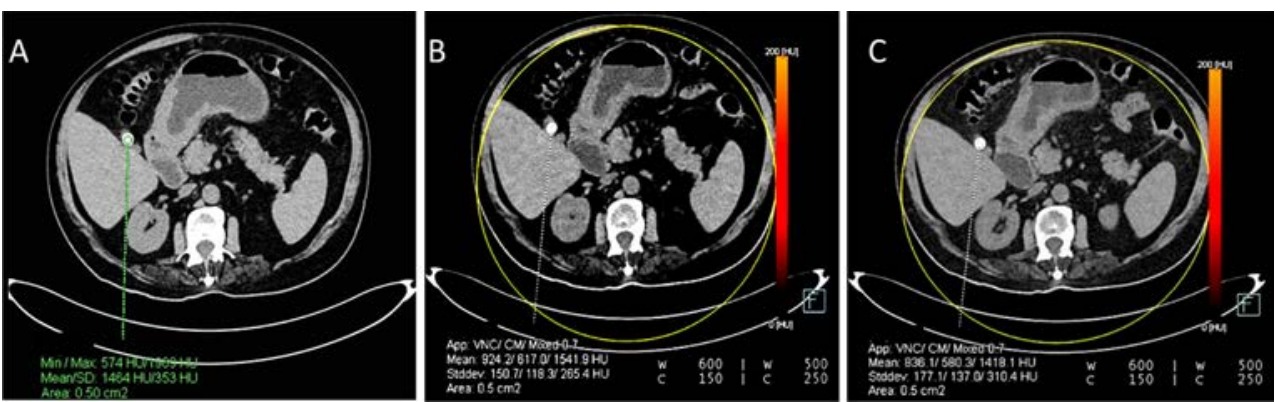

Fig. 4 Attenuation of gallbladder calculus in (A) noncontrast unenhanced image (B) arterial virtual unenhanced image (C) portal venous virtual unenhanced image. 

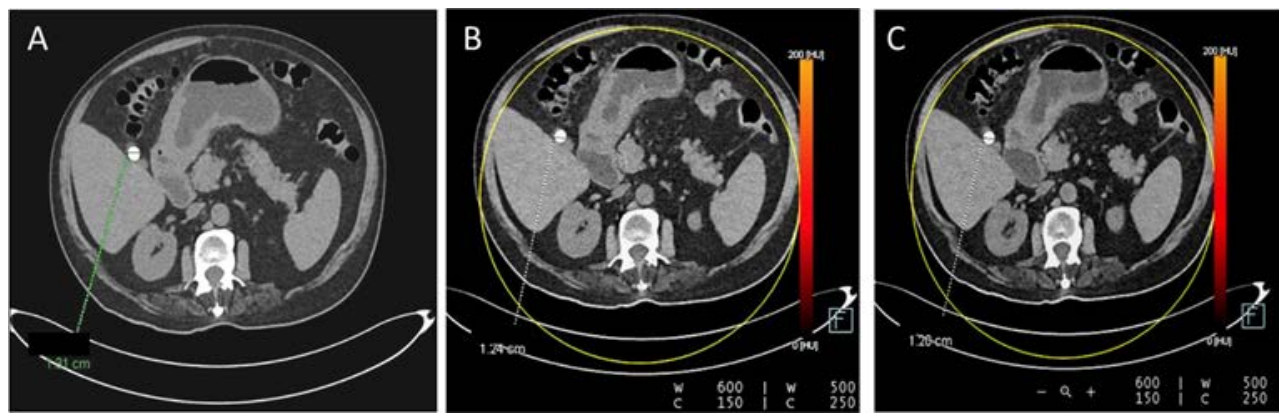

Fig. 5 Size of gallbladder calculus in (A) noncontrast unenhanced image (B) arterial virtual unenhanced image (C) portal venous virtual unenhanced image.

size and $\mathrm{HU}$ in VUI when compared with true noncontrast images. ${ }^{20}$ Hence, CUI should be preferred over VUI in evaluation of patients with suspected biliary colic or vascular pathologies.

Out of 60 patients in our study, 24 patients had parenchymal lesions. Eighteen of them had solid lesions and the rest had cystic lesions. The parenchymal lesions include hepatocellular carcinoma (HCC), metastasis, abscess, cysts, and calcified granuloma in the liver, angiomyolipoma, cyst in the pancreas, and GB carcinoma. The mean HU of all the lesions detected during the scan in CUI and VUI were analyzed using ANOVA and the $p$-value of 0.8 was obtained, which was found to be insignificant, indicating VUI were comparable to CUI in lesion detection and characterization. Also, we separated the enhancing noncalcified solid lesions and cystic lesions separately and did a statistical analysis. The $p$-value for difference in mean attenuation was insignificant in both the solid and cystic groups and measured 0.82 and 0.58 , respectively, indicating that VUI were comparable with true noncontrast images ( - Figs. 6 and $\mathbf{7}$ ). There were two lesions with calcification within whose $\mathrm{HU}$ values were underestimated in both virtual unenhanced datasets when compared with the noncontrast image. This is corresponding to the previous study done by De Ceccoa et al, in which they stated that VUI were superior to noncontrast images in detecting hypoattenuating lesions and inferior in detecting calcified lesions. ${ }^{12}$

In our study, all the VUI were of score 3 or more, indicating that they were suitable for interpretation. The individual difference between the mean score was statistically significant with $p$-value $<0.01$. This indicates that the arterial VUI is statistically superior in image quality when compared with the portal venous VUI. This result is similar to the study done by De Ceccoa et al, which showed that AVU were slightly superior to PVU in lesion detection. ${ }^{12}$ This can be explained by the fact that in arterial phase, contrast has not filled in the interstitial space completely and can be washed off easily. However, in a study done by Lehti et al, they have concluded that PVU images are superior than AVU images and better approximate
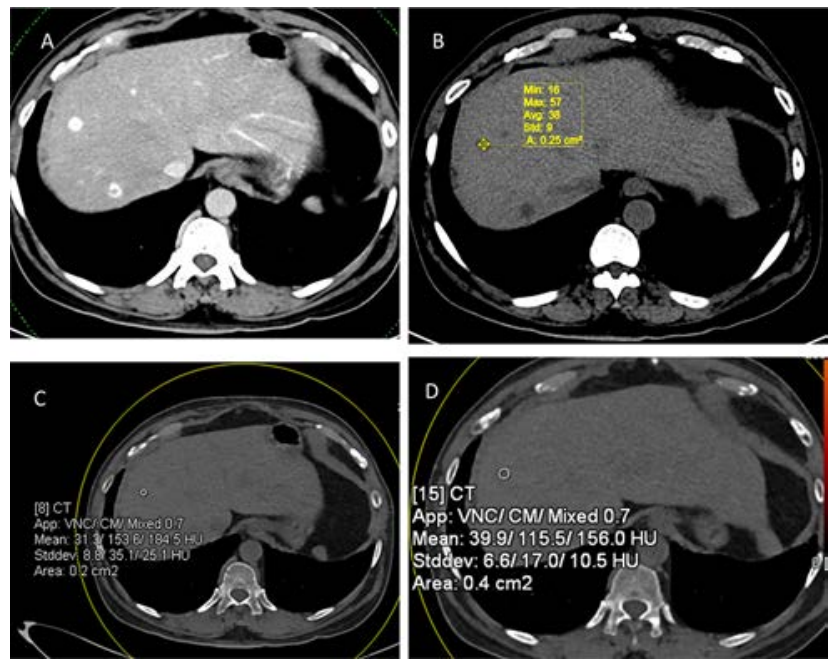

Fig. 6 Axial CT image (A) of a patient with history of carcinoma thyroid shows hypervascular liver lesions suggestive of metastasis. Attenuation of the lesion in noncontrast unenhanced image (B), arterial virtual unenhanced image $(\mathbf{C})$, and portal venous virtual unenhanced image (D) shows no significant difference.
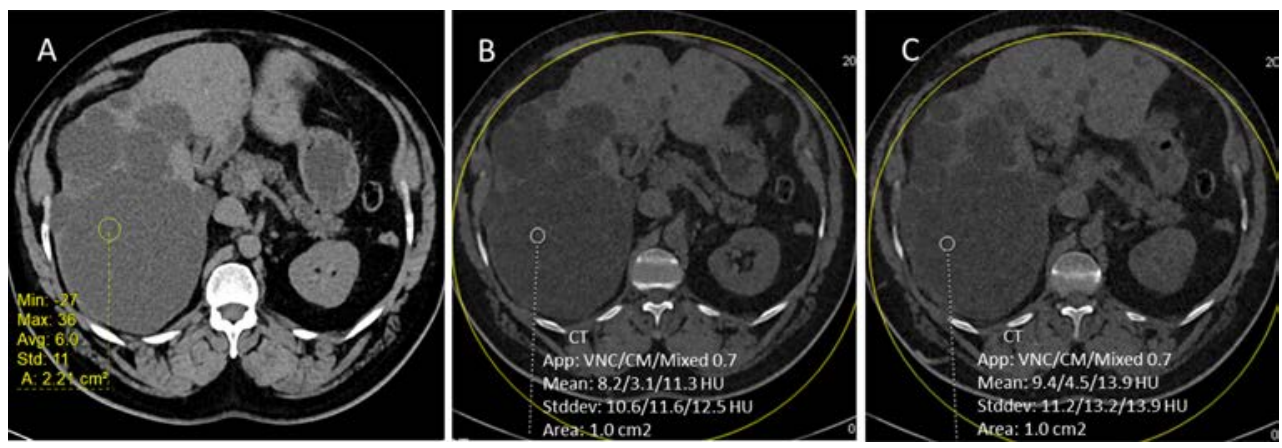

Fig. 7 Axial plain CT image (A) shows multiple cystic lesions of varying sizes in liver. Attenuation of cystic lesion in the right lobe of the liver in (A) noncontrast unenhanced image, (B) arterial virtual unenhanced image, (C) and portal venous virtual unenhanced image shows no significant difference. 
noncontrast unenhanced (NCU) images. ${ }^{21}$ The possible reasons could be that their study was done in second-generation, dual-source CT and they have started their arterial phase acquisition earlier compared with our scans. Complete separation of iodine signal is possible in third-generation, dual-source CT because of its advanced hardware and software applications.

We found in our study that by eliminating conventional unenhanced acquisition from triple-phase abdominal imaging and by substituting it with virtual noncontrast $\mathrm{CT}$, we can achieve an average ED reduction of $39 \%$. The results were similar to the few studies described in previous literature. ${ }^{11,12,22}$

There were few limitations in our study. This was a single institutional study with limited subjects. This study was done on the dual-source, dual-energy technique. Hence, the virtual unenhanced values obtained cannot be generalized to other techniques of DECT like rapid kilovolt switching, multilayer detector technique, etc. This study was done using the contrast agent omnipaque (iohexol) with a flow rate of $4 \mathrm{~mL} / \mathrm{sec}$. The other iodinated contrast agents like iopamidol and iodixonal as well as the other flow rates used in other protocols were not evaluated. We have evaluated the attenuation values of only the liver, pancreas, spleen, aorta, IVC, and MPV. The other organs were not included in this study. The lesions were grouped into cystic and solid lesions and then analyzed; however, individual lesions like HCC, metastasis, etc. were not statistically analyzed due to their small numbers. Some of the other pathologies like hematoma, pancreatitis, and associated collection were also not evaluated, as we did not encounter these pathologies in our study. With the paucity of available studies, further studies are necessary to fully evaluate the potential of virtual unenhanced images and to make it a standard of care.

\section{Conclusion}

Virtual unenhanced images generated in the third-generation, dual-source, dual-energy CT possesses diagnostic image quality and can replace the conventional unenhanced images in triple-phase studies done for the hepatopancreaticobiliary system. In our study, the virtual unenhanced image obtained from arterial phase is superior to those obtained from portal venous phase. A mean ED reduction of 39\% can be achieved by eliminating conventional unenhanced acquisition.

\section{Conflict of Interest}

None declared.

\section{References}

1 Johnson TR. Dual-energy CT: general principles. AJR Am J Roentgenol 2012;199(5(Suppl):S3-S8

2 Coursey CA, Nelson RC, Boll DT, et al. Dual-energy multidetector CT: how does it work, what can it tell us, and when can we use it in abdominopelvic imaging? Radiographics 2010;30(4):1037-1055

3 Raptopoulos V, Karellas A, Bernstein J, Reale FR, Constantinou C, Zawacki JK. Value of dual-energy CT in differentiating focal fatty infiltration of the liver from low-density masses. AJR Am J Roentgenol 1991;157(4):721-725
4 Silva AC, Morse BG, Hara AK, Paden RG, Hongo N, Pavlicek W. Dual-energy (spectral) CT: applications in abdominal imaging. Radiographics 2011;31(4):1031-1046, discussion 1047-1050

5 Mileto A, Mazziotti S, Gaeta M, et al. Pancreatic dual-source dual-energy CT: is it time to discard unenhanced imaging? Clin Radiol 2012;67(4):334-339

6 Johnson TR, Krauss B, Sedlmair M, et al. Material differentiation by dual energy $\mathrm{CT}$ : initial experience. Eur Radiol 2007;17(6):1510-1517

7 Schenzle JC, Sommer WH, Neumaier K, et al. Dual energy CT of the chest: how about the dose? Invest Radiol 2010;45(6):347-353

8 Solomon J, Mileto A, Ramirez-Giraldo JC, Samei E. Diagnostic performance of an advanced modelled iterative reconstruction algorithm for low contrast detectability with a third generation dual source multidetector CT scanner: potential for radiation dose reduction in a multireader study. Radiology 2015;275(3):735-745

9 De Cecco CN, Buffa V, Fedeli S, et al. Dual energy CT (DECT) of the liver: conventional versus virtual unenhanced images. Eur Radiol 2010;20(12):2870-2875

10 Gordic S, Desbiolles L, Stolzmann P, et al. Advanced modelled iterative reconstruction for abdominal CT: qualitative and quantitative evaluation. Clin Radiol 2014;69(12):e497-e504

11 Li Y, Li Y, Jackson A, et al. Comparison of virtual unenhanced $\mathrm{CT}$ images of the abdomen under different iodine flow rates. Abdom Radiol (NY) 2017;42(1):312-321

12 De Cecco CN, Muscogiuri G, Schoepf UJ, et al. Virtual unenhanced imaging of the liver with third-generation dual-source dual-energy CT and advanced modeled iterative reconstruction. Eur J Radiol 2016;85(7):1257-1264

13 Chiro GD, Brooks RA, Kessler RM, et al. Tissue signatures with dual-energy computed tomography. Radiology 1979;131(2):521-523

14 Millner MR, McDavid WD, Waggener RG, Dennis MJ, Payne WH, Sank VJ. Extraction of information from CT scans at different energies. Med Phys 1979;6(1):70-71

15 Kalender WA, Perman WH, Vetter JR, Klotz E. Evaluation of a prototype dual-energy computed tomographic apparatus. I. Phantom studies. Med Phys 1986;13(3):334-339

16 Kelcz F, Joseph PM, Hilal SK. Noise considerations in dual energy CT scanning. Med Phys 1979;6(5):418-425

17 Graser A, Johnson TR, Hecht EM, et al. Dual-energy CT in patients suspected of having renal masses: can virtual nonenhanced images replace true nonenhanced images? Radiology 2009;252(2):433-440

18 Durieux P, Gevenois PA, Muylem AV, Howarth N, Keyzer C. Abdominal attenuation values on virtual and true unenhanced images obtained with third-generation dual-source dual-energy CT. AJR Am J Roentgenol 2018;210(5):1042-1058

19 Laukamp KR, Ho V, Obmann VC, et al. Virtual non-contrast for evaluation of liver parenchyma and vessels: results from 25 patients using multi-phase spectral-detector CT. Acta Radiol 2019;0(0):1-10

20 Lee HA, Lee YH, Yoon KH, Bang DH, Park DE. Comparison of virtual unenhanced images derived from dual-energy ct with true unenhanced images in evaluation of gallstone disease. AJR Am J Roentgenol 2016;206(1):74-80

21 Lehti L, Söderberg M, Höglund P, Wassélius J. Comparing arterial- and venous-phase acquisition for optimization of virtual noncontrast images from dual-energy computed tomography angiography. J Comput Assist Tomogr 2019;43(5):770-774

22 Kaza RK, Raff EA, Davenport MS, Khalatbari S. Variability of CT Attenuation measurements in virtual unenhanced images generated using multimaterial decomposition from fast kilovoltage-switching Dual-energy CT. Acad Radiol 2017;24(3):365-372 